\title{
Introduction to the focused section on machine learning, estimation and control for intelligent robotics
}

\author{
Jiong Tang ${ }^{1} \cdot \mathrm{Xu}_{\mathrm{Chen}}{ }^{2} \cdot \operatorname{Yan} \mathrm{Gu}^{3} \cdot$ Minghui Zheng $^{4}$
}

Published online: 5 November 2020

(c) Springer Nature Singapore Pte Ltd. 2020

Owing to the explosive advancements in recent years in the areas of computational intelligence, material synthesis, and device integration, a new era of intelligent robotics, featuring unprecedented capabilities of sensing, actuation, and decision making, has arrived. Various newly developed robotic systems have penetrated into virtually all industrial sectors, ranging from manufacturing, energy, aerospace and naval, infrastructure, to health care and service, etc. They possess remarkably enhanced performances in terms of accuracy, adaptivity, reliability, and autonomy, and are poised to change fundamentally our modalities of working and living. The new accomplishments exemplify the collaborative efforts by academia and industry that are currently accelerating.

Aiming at documenting and disseminating the progresses and identifying growth opportunities, this "Focused Section on Machine Learning, Estimation and Control for Intelligent Robotics" of the International Journal of Intelligent Robotics and Applications (IJIRA) showcases a number of recent technological achievements in the learning and control of robotic systems. It includes nine papers that share the common theme of robotic systems utilizing complex or heterogeneous data in their design and control through advanced learning and estimation techniques.

The paper "Robotic Target Following with Slow and Delayed Visual Feedback", by Xiao and Chen, provides a control method for autonomously tracking moving targets

Jiong Tang

jiong.tang@uconn.edu

1 Department of Mechanical Engineering, University of Connecticut, Storrs 06269, USA

2 Department of Mechanical Engineering, University of Washington, Seattle, WA 98195, USA

3 Department of Mechanical Engineering, University of Massachusetts Lowell, Lowell, MA 01854, USA

4 Department of Mechanical and Aerospace Engineering, University at Buffalo, Buffalo, NY 14260, USA with slow and delayed visual feedback. Such capabilities underpin robotic applications ranging from transportation to medical systems. Compared to classic position-based visual servo, the proposed approach achieves $95 \%$ reduction of tracking errors when partial knowledge of the target dynamics is known. The result is achieved via a tripartite approach including (1) a velocity control of the robot that ensures exponential reduction of the visual feature error even subject to a maneuvering target, (2) a pose and velocity estimation that considers the dynamics of the moving target, and (3) a multirate information recovery that recovers fast motion estimates from slow and delayed visual feedback.

In "Handling Crowdsourced Data using State Space Discretization for Robot Learning and Synthesizing Physical Skills", Zhao et al. consider the problem of continuous robot learning towards ubiquitous robot physical intelligence. In view of the emerging opportunities in machine learning from large datasets, the authors propose to replicate the success of crowdsourced human computation in robot physical intelligence. Sensorimotor information from a group of teleoperators is collected. The paper then uses a state-space discretization to manage the data and synthesize robot skills. Two types of algorithms are proposed and justified via case studies pertaining to robotic object manipulation.

In "Influence of Human Operator on Stability of Haptic Rendering: A Closed-Form Equation”, Mashayekhi et al. present a new formulation for stability analysis of rigid haptic devices in presence of a human operator. This formulation enables the prediction of stability boundaries within large parametric ranges of time delay and virtual damping. The formulation can relate all parameters of the operator's hand, virtual environment, haptic device, sampling time, and time delay in a unified equation. The influence of the operator's hand and effective mass on stability is studied analytically and verified by simulations and experiments on a KUKA lightweight robot.

In "Error Modeling and Sensitivity Analysis of a Parallel Robot with R-(SS) ${ }^{2}$ Branches", Huang et al. investigate 
the error model of parallel robots with R-(SS) ${ }^{2}$ (R, actuated revolute joint; $S$, spherical joint) branches that are widely used in high-speed sorting. By considering the error transmission mode of the dual-moving platform as a common straight line, a general form of the error model containing all possible geometric errors is established. The sensitive geometric errors are then identified. It is demonstrated that reducing the geometric errors with relatively higher sensitivity values has better effect. The simulation results show that both larger volumetric errors and rotational errors tend to aggregate near the boundaries of the workspace. This improved error modeling method takes the adaptability of the error modeling into account, and can be extended to the specific error modeling of the corresponding robot.

In "Shape Estimation for Elongated Deformable Object using B-spline Chained Multiple Random Matrices Model", Yao et al. propose a B-spline chained multiple random matrix model (RMM) representation to model geometric characteristics of an elongated deformable object. This shape estimation problem is challenging due to the hyper degrees of freedom structure of the elongated deformable object. Based on the likelihood function of the proposed B-spline chained multiple RMM, an expectation-maximization method is derived to estimate the shape of the elongated deformable object. A split and merge method based on the Euclidean minimum spanning tree is proposed to provide initialization for the EM algorithm. The performance of the proposed shape estimation algorithm is evaluated using real measurements of a red dock line in 6 different configurations. The experimental results demonstrate that the B-spline curve chained RMMs algorithm is capable of estimating the shape of the elongated deformable object configured as intersecting and non-intersecting shapes.

The paper "A Recurrent Neural Network for Variable Admittance Control in Human-Robot Cooperation: Simultaneously and online Adjustment of the Virtual Damping and Inertia parameters", by Sharkawy et al., introduces a recurrent neural network for variable admittance control in human-robot cooperation tasks for optimal adjustment of both the virtual damping and the virtual inertia coefficients simultaneously based on online Jordan recurrent neural network (JRNN) training. This network is indirectly trained using the real-time recurrent learning algorithm and based on the velocity error between the reference velocity of the minimum jerk trajectory model and the actual velocity of the robot. The generalization and the effectiveness of the proposed method are illustrated by multiple subjects testing through comparisons with existing methods.

In "Adaptive Formation-Switching of a Multi-Robot System in an Unknown Occluded Environment using BAT Algorithm", Roy et al. propose an adaptive formation control strategy which enables the robots to navigate through the landscape without crashing into obstacles, while maintaining a strong inter-agent cohesiveness and simultaneously switching their formation when situations change. The formation control law is designed based on the echolocation principle of the bio-inspired bat algorithm, and rests on three components: attraction, repulsion, and obstacle avoidance controllers. Extensive simulation and experimental studies have been conducted to validate the proposed scheme.

The paper "Vehicle Specific Robust Traversability Indices Using Roadmaps on 3D Pointclouds" by Khan et al. provides a framework to determine whether a particular segment of the road is traversable for a particular vehicle, by considering the obstacle size, number, and locations. Two indexes, including the Road Traversability Index and the Road Safety Index, have been introduced and analyzed. The proposed analysis framework takes terrain (road and obstacles), vehicle parameters, motion model, and collision criterion into consideration, and leverages the graph-theory approach to infer traversability. This framework has been applied to various real-world scenarios, and it shows the proposed roadmap-based traversability can be used to assess the safety of the road for a particular vehicle.

Lastly, in "Robust State Dependent Riccati Equation Variable Impedance Control for Robotic Force-Tracking Tasks", Roveda and Piga focus on the derivation and validation of a sensorless model-based methodology for high-performance force control demanded by various human-robot interaction applications. Based on sensorless Cartesian impedance control, an Extended Kalman Filter (EKF) has been designed to estimate the physical interaction between the robot and the environment. Such estimation is then used to close a robust high-performance force loop designed by exploiting a variable impedance controller and a State Dependent Riccati Equation (SDRE) force controller. This approach has been validated through simulations on a Franka EMIKA panda robot for a probing task involving materials with different stiffnesses. Simulation results demonstrate the effectiveness of the proposed method in guaranteeing enhanced stability and tracking performance compared with an LQR controller.

We sincerely thank the IJIRA and its Editor-in-Chief, Professor Kok-Meng Lee, for giving us the opportunity to serve as guest editors for this Focused Section. We appreciate the great efforts by all the authors submitting interesting and cutting-edge papers, and acknowledge the contribution by the anonymous reviewers for providing rigorous assessments. We also thank the managing staff of the IJIRA for meticulous assistance throughout the production of this Focused Section.

Publisher's Note Springer Nature remains neutral with regard to jurisdictional claims in published maps and institutional affiliations. 


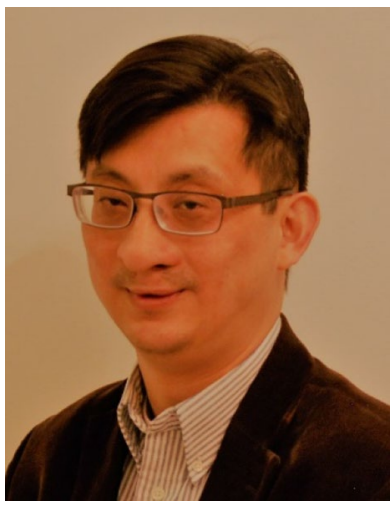

Jiong Tang received the B.S. and M.S. degrees in Applied Mechanics from Fudan University, China, in 1989 and 1992, respectively, and the Ph.D. degree in Mechanical Engineering from the Pennsylvania State University, USA, in 2001. He worked at the GE Global Research Center as Mechanical Engineer during 2001 to 2002. He then joined the Mechanical Engineering Department, University of Connecticut where he is currently a Professor. His research interests encompass dynamical systems, sensing, control, and computational intelligence with applications to manufacturing, energy, aerospace, and infrastructure systems. He has been involved in a large number of research projects with funding totaling over \$20 M. Dr. Tang has served as Associate Editor for IEEE/ASME Transactions on Mechatronics, IEEE Transactions on Instrumentation and Measurement, ASME Journal of Vibration and ASME Journal of Dynamic Systems, Measurement, and Control. He is a fellow of ASME.

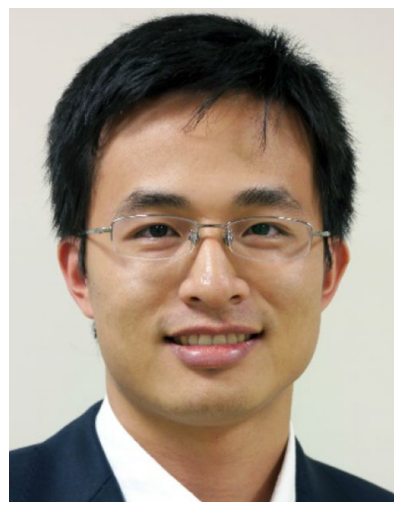

Xu Chen is an assistant professor in the Department of Mechanical Engineering at the University of Washington (UW), Seattle. He received his M.S. and Ph.D. degrees in Mechanical Engineering from the University of California, Berkeley in 2010 and 2013, respectively, and his bachelor's degree with honors from Tsinghua University, China in 2008. He leads the MACS Lab-a team focusing on sensing, controls, and automation that facilitate, e.g., robots that inspect complex, highly reflective parts in aerospace, and additive manufacturing that makes engineered materials with extraordinary properties at low unit costs compared to conventional machining. Xu Chen's work has led to Best Paper Awards, servo algorithms in industrial mass production in the information storage industry, top-tier adaptive control methods in international benchmark evaluations, and the graduation of two University Scholars.
Dr. Chen is a recipient of the U.S. National Science Foundation CAREER Award. Since 2019, he has been serving in the Scientific Advisory Committees of the Boeing Advanced Research Center and the UW Advanced Composite Center.

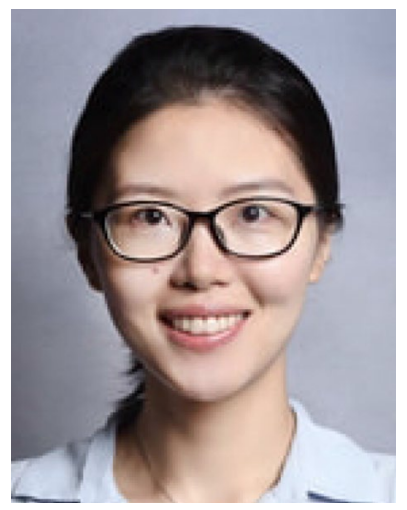

Yan Gu received her B.S. degree in Mechanical Engineering from Zhejiang University (China) in June 2011 and her Ph.D. degree in Mechanical Engineering from Purdue University in August 2017. She was the recipient of the Chu Kochen Scholarship at Zhejiang University, and the Frederick N. Andrews Fellowship and the Estus H. and Vashti L. Magoon Award for Excellence in Teaching at Purdue University. She joined the Department of Mechanical Engineering at the University of Massachusetts Lowell as an Assistant Professor in September 2017. Her research interests include nonlinear control, hybrid systems, legged locomotion, wearable robots, and human biomechanics. Dr. Gu's work has received the 2019 Verizon's 5G Robotics Challenge Award and has been recognized as the Best Student Paper Finalist at 2020 IEEE/ASME International Conference on Advanced Intelligent Mechatronics. She is currently an Associate Editor of IEEE-RAS Robotics and Automation Letters.

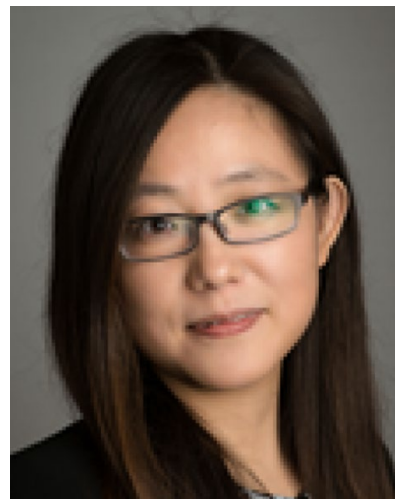

Minghui Zheng received the B.E. and M.E. degrees, in 2008 and 2011 respectively, from Beihang University, Beijing, China, and the Ph.D. degree in Mechanical Engineering, in 2017, from the University of California, Berkeley, USA. She joined University at Buffalo, NY, USA, in 2017, where she is currently an assistant professor in the Department of Mechanical and Aerospace Engineering. Her research interests include learning and control with applications to unmanned aerial vehicles for disaster resilience and collaborative robots for manufacturing. 